\title{
Channel Prediction for Adaptive Modulation in Underwater Acoustic Communications
}

\author{
Andreja Radosevic ${ }^{\dagger}$, Tolga M. Duman ${ }^{\dagger \dagger}$, John G. Proakis ${ }^{\dagger}$, and Milica Stojanovic ${ }^{\ddagger}$ \\ ${ }^{\dagger}$ Dept. of Electrical and Computer Engineering, Univ. of California, San Diego, La Jolla, CA 92093 \\ ${ }^{\dagger}$ School of Electrical, Computer and Energy Engineering, Arizona State University, Tempe, AZ 85287-5706 \\ ${ }^{\ddagger}$ Dept. of Electrical and Computer Engineering, Northeastern University, Boston, MA 02115
}

\begin{abstract}
In this paper we explore the feasibility of predicting an underwater acoustic (UWA) channel impulse response one travel time ahead. Our work is motivated by the design of UWA communication systems that will employ adaptive modulation, whose performance relies on the ability to predict the channel up to a few seconds into the future. The possibility of predicting the channel response is demonstrated using real data collected during an experiment conducted in shallow water off the western coast of Kauai, Hawaii, in June 2008. The key to enabling prediction is decoupling of the effects of motion-induced phase offset from the more slowly varying channel path gains. It is shown that a significant performance improvement can be achieved using prediction as compared to using outdated channel estimates.
\end{abstract}

\section{INTRODUCTION}

Underwater acoustic (UWA) channels are generally characterized by randomly time-varying multipath propagation which results in frequency-selective fading [1]. A typical UWA channel exhibits long delay spreads $(10-100 \mathrm{~ms})$; however, the channel impulse response often has a sparse structure, with only a few propagation paths carrying the significant portion of channel energy.

Time-varying multipath propagation and limited bandwidth put significant constraints on the achievable throughput for UWA communications. In order to increase the bandwidth efficiency of UWA systems, adaptive modulation schemes can be employed. Although various adaptive signaling techniques have been extensively applied to radio channels [2][3], not much has been done in the area of UWA communications [4].

In this paper, we explore the feasibility of predicting the impulse response of a shallow water UWA channel. A similar possibility was discussed in [5]. Our ultimate goal is to design adaptive modulation methods for UWA communication systems in which the channel impulse response characteristic, i.e. the channel state information (CSI), is measured at the receiver and fed back to the transmitter. Since sound propagates at a very low speed (nominally $1500 \mathrm{~m} / \mathrm{s}$ ), the design of an adaptive system relies on the ability to predict the channel at least one travel time ahead. This is a very challenging task for communications in the range of several kilometers.

Our approach is the following: first we estimate small Doppler rates (less then $10^{-4}$ ) that correspond either to drifting of the instruments, or residuals after initial resampling in

This work is supported by the Multidisciplinary University Research Initiative (MURI) ONR Grants N00014-07-1-0738 and N00014-07-1-0739. mobile systems (e.g. systems using autonomous underwater vehicles). Proper Doppler compensation ensures stability over intervals of time that are long enough to support channel prediction several seconds ahead. We then exploit the sparse multipath structure of the channel to simplify the prediction problem. Specifically, we estimate only a few significant paths of the channel within a possibly large overall delay spread. Finally, we treat the statistical properties of the underlying random process of the channel fading as unknown, and compute the parameters of a linear predictor adaptively, by applying the recursive least-squares (RLS) algorithm [6]. Since the channel varies during propagation [7], we implement an RLS algorithm using a low order with a small forgetting factor. Moreover, because multiple paths may be correlated, we investigate their joint prediction. We employ multi-channel processing to further improve channel prediction by combining the signals received over an array of hydrophones. The complexity of the resulting algorithm remains low since only a small number of significant channel paths are predicted, and the predictor order is low. Using experimental data, we demonstrate that the proposed method is capable of providing reliable CSI to the transmitter.

The paper is organized as follows. In Section II, we describe the model that characterizes an UWA channel. In Section III, we introduce a linear RLS predictor for the channel tap coefficients. In Section IV, we present experimental results that demonstrate the performance of the predictor. In Section V, we present our concluding remarks.

\section{Channel Model}

Let us define the impulse response of the channel as

$$
h(\tau, t)=\sum_{p=0}^{P-1} h_{p}(t) \delta\left(\tau-\tau_{p}(t)\right)
$$

where $P$ is the number of distinct propagation paths, $\tau$ is the delay variable and $t$ is the time at which the channel is observed. The coefficient $h_{p}(t)$ represents the real-valued gain of the $p$-th path, and $\tau_{p}(t)$ represents the corresponding delay. This impulse response links the transmitted signal $s(t)$ and the received signal $r(t)$ as

$$
r(t)=\sum_{p=0}^{P-1} h_{p}(t) s\left(t-\tau_{p}(t)\right)+n(t)
$$


where $n(t)$ represents additive white Gaussian noise. If we also define the equivalent baseband signals $u(t)$ and $v(t)$ with respect to a frequency $f_{c}$, such that

$$
\begin{gathered}
s(t)=\operatorname{Re}\left\{u(t) e^{j 2 \pi f_{c} t}\right\} \\
r(t)=\operatorname{Re}\left\{v(t) e^{j 2 \pi f_{c} t}\right\} .
\end{gathered}
$$

We then obtain

$$
v(t)=\sum_{p=0}^{P-1} c_{p}(t) u\left(t-\tau_{p}(t)\right)+w(t),
$$

where

$$
c_{p}(t)=h_{p}(t) e^{-j 2 \pi f_{c} \tau_{p}(t)},
$$

and $w(t)$ is the equivalent baseband noise. Expression (4) implies the equivalent baseband channel response

$$
c(\tau, t)=\sum_{p=0}^{P-1} c_{p}(t) \delta\left(\tau-\tau_{p}(t)\right) .
$$

\section{A. Modeling of the time-varying path delay $\tau_{p}(t)$}

We model the time-varying path delays as

$$
\tau_{p}(t)=\tau_{p 0}-\int_{x=0}^{t} a_{p}(x) d x .
$$

This model includes the fixed term $\tau_{p 0}$ which describes the propagation delay corresponding to the system geometry at the time of transmission, and an additional term $\int_{x=0}^{t} a_{p}(x) d x$ that describes the effect of motion at the time of observation. The system motion during a period of time corresponding to a few seconds (or several data packets) is modeled by velocity and acceleration terms ${ }^{1}$ which lead to a linear Doppler rate $a_{p}(t)$. Specifically, we model $a_{p}(t)$ as a piecewise linear function

$$
a_{p}(t)=a_{p}[n-1]+\left(a_{p}[n]-a_{p}[n-1]\right)\left(\frac{t}{T}-n+1\right),
$$

where $(n-1) T \leq t \leq n T$, and $a_{p}[n]$ are the Doppler scaling factors evaluated at time instances $n T$. This model is deemed suitable for the time scales of interest to an adaptive UWA communication system, since channel prediction depends on the availability of a stable signal reference that can be obtained through accurate motion compensation. For example, for a $4 \mathrm{~km}$ link and a carrier frequency $f_{c}=16 \mathrm{kHz}$, a small Doppler rate $\left|a_{p}(t)\right| \leq 1.2 \cdot 10^{-5}$ can cause the phase of $c_{p}(t)$ in Eq. (5) to change up to $\pi$ radians, during a time interval of 2.67 seconds that corresponds to the propagation delay of one travel time. ${ }^{2}$ Such a phase shift can seriously degrade the performance of channel prediction.

The model (7) allows one to decouple the phase $2 \pi f_{c} \tau_{p}(t)$ into two terms, one that is not related to motion, and another that is related to motion. While the first term may not be

\footnotetext{
${ }^{1}$ A more accurate model could include higher-order terms; however, experimental results confirm that this is not necessary.

${ }^{2}$ Here we should make a distinction between making the prediction for one travel time ahead, and for the round-trip time (two travel times ahead), since the two cases correspond to different feedback implementation strategies, i.e. different functions performed by the two ends of a link.
}

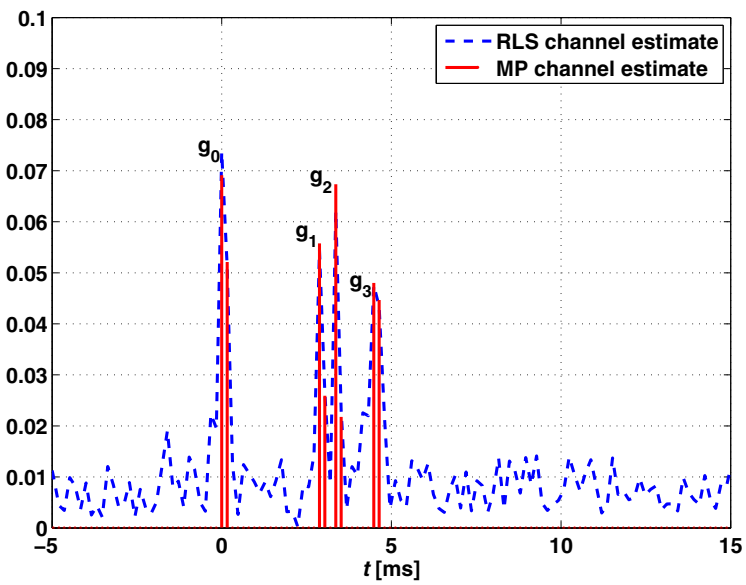

Fig. 1. Channel estimates obtained by the RLS and the MP algorithm.

predictable with sufficient accuracy because the frequency $f_{c}$ may be many times greater than the inverse of the path delay, the second term can be predicted using the estimates of the Doppler scaling factors $a_{p}[n]$. With this in mind, we proceed to develop a channel prediction method that focuses on two general terms: a complex-valued coefficient $g_{p}(t)=h_{p}(t) e^{-j 2 \pi f_{c} \tau_{p 0}}$, and a motion-induced phase $\theta_{p}(t)=$ $2 \pi f_{c} \int_{x=0}^{t} a_{p}(x) d x$. In other words, we model the baseband channel response as

$$
c(\tau, t)=\sum_{p=0}^{P-1} g_{p}(t) e^{-j \theta_{p}(t)} \delta\left(\tau-\tau_{p}(t)\right)
$$

where we treat each $g_{p}(t)$ as an unknown complex-valued channel coefficient, which is assumed to be stable over a prolonged period of time (tens of seconds), and $\theta_{p}(t)$ as an unknown motion-induced phase, which is modeled as a second-order polynomial based on the expressions (7) and (8). We emphasize that this model is valid for some interval of time, but may change from one such interval to another.

Our goal is to develop a two-step procedure in which we first estimate the channel coefficients at the receiver from a probe signal, and then use the so-obtained estimates to form predictions, which are finally sent back to the transmitter. Below, we address each of these steps separately.

\section{B. Channel estimation}

Channel estimation consists of two steps. In the first step, phase compensation is performed to produce a stable reference signal. In the second step, the so-obtained signal is used to estimate the path coefficients $g_{p}(t)$. The process relies on the estimates of the Doppler scaling factors $a_{p}[n]$, which are assumed to be available with a certain precision. The Doppler factors are not needed thereafter, as we conjecture that the channel coefficients after motion-compensation exhibit sufficient stability to allow prediction several seconds into the future. 

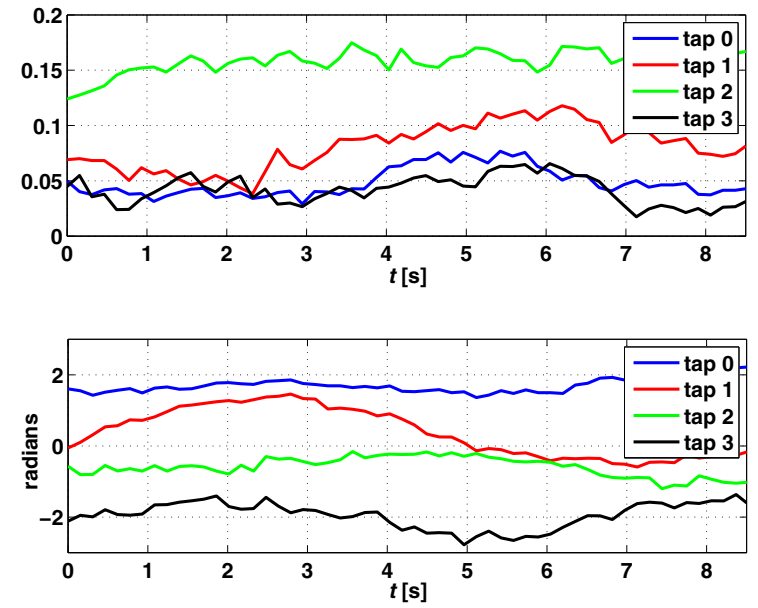

Fig. 2. Magnitudes (top) and phases (bottom) of the channel path coefficients.

Fig. 1 illustrates the channel estimates obtained from real data collected during the Kauai Acomms MURI 2008 (KAM08) experiment, which was conducted in shallow water off the western coast of Kauai, Hawaii, in June 2008. After the initial phase compensation, we perform channel estimation from the received signal using the matching pursuit (MP) algorithm [8]. Note from Fig. 1 that the MP algorithm produces 8 coefficients, where neighboring coefficients belong to the same propagation path due to the path dispersion [1]. For further analysis we merge adjacent coefficients so as to represent four propagation paths $g_{0}, g_{1}, g_{2}$ and $g_{3}$. Therefore, the MP algorithm provides estimates of the channel coefficients $g_{p}(t)$, assuming that $P=4$ channel coefficients are sufficient for the description of the sparse multipath structure. These estimates are denoted by $\tilde{g}_{p}[n]$, and computed at time instances $n T$ separated by $T=155 \mathrm{~ms}$. For comparison purposes, we also provide the channel estimate obtained using the RLS algorithm. Different peaks in the channel estimates can be associated with the multiple surface and bottom reflections calculated from the geometry of the experiment. As it can be seen from the figure, the MP algorithm successfully estimates the significant channel coefficients, and reduces the estimation error with respect to the RLS.

We emphasize that positions of the significant paths may drift on a larger time scale (tens of seconds), and therefore have to be updated accordingly. In Fig. 2, we show the magnitudes and phases of the significant paths over a time period of $8 \mathrm{~s}$. As we initially conjectured, the phases of $g_{p}(t)$ remain relatively stable for more than a few seconds (a propagation delay over several kilometers).

\section{ChanNEL PREDICTION}

The future values of $g_{p}(t)$ are predicted from the estimates $\tilde{g}_{p}(t)$. We assume that $L$ receive elements are available, and we combine their observations to exploit a multi-channel processing gain. Specifically, we use $M$ observations made at times $n, n-1, \ldots, n-M+1$ to predict the channel at time $n+N$. To account for possible correlation between the path coefficients, we allow for their joint prediction. In other words, we use all $P$ channel coefficients from each of the $L$ different receive elements to predict each new coefficient. The prediction is thus made as

$$
\hat{\mathbf{g}}[n+N]=\mathbf{W}^{H}[n] \tilde{\mathbf{g}}_{M L}[n],
$$

where

$$
\begin{aligned}
& \hat{\mathbf{g}}[n+N]=\left[\begin{array}{llll}
\tilde{g}_{0}[n+N] & \tilde{g}_{1}[n+N] \ldots & \left.\tilde{g}_{P-1}[n+N]\right]^{T},
\end{array}\right. \\
& \tilde{\mathbf{g}}_{M L}[n]=\left[\begin{array}{llll}
\tilde{\mathbf{g}}_{M}^{0}{ }^{T}[n] & \ldots & \tilde{\mathbf{g}}_{M}^{1}{ }^{T}[n] & \left.\tilde{\mathbf{g}}_{M}^{L-1 T}[n]\right]^{T},
\end{array}\right.
\end{aligned}
$$

and

$$
\begin{aligned}
\tilde{\mathbf{g}}_{M}^{l}[n]= & {\left[\tilde{g}_{0}^{l}[n] \ldots \tilde{g}_{0}^{l}[n-M+1] \tilde{g}_{1}^{l}[n] \ldots\right.} \\
& \left.\tilde{g}_{1}^{l}[n-M+1] \tilde{g}_{P-1}^{l}[n] \ldots \tilde{g}_{P-1}^{l}[n-M+1]\right]^{T},(
\end{aligned}
$$

for $0 \leq l \leq L-1$. The matrix $\mathbf{W}[n]$ contains $M L P \times P$ prediction coefficients that are to be determined.

Because the second-order statistics are not available for the random process $\mathbf{g}(n+N)$, we compute $\mathbf{W}(n)$ adaptively, by applying the RLS algorithm specified in Table I.

In Eq. (15), $\mathbf{R}$ is an $M L P \times M L P$ matrix which represents an estimate of the inverse joint auto-correlation matrix $E\left\{\tilde{\mathbf{g}}_{M L}[n] \tilde{\mathbf{g}}_{M L}^{H}[n]\right\}$ and $\delta$ is a small constant, typically a fraction of the minimum among variances of the channel coefficients jointly predicted by the RLS algorithm.

TABLE I

\begin{aligned} \hline \hline & The algorithm initialization: \\ $\mathbf{W}[0]= & \mathbf{0} \\ \mathbf{R}[0]= & \delta^{-1} \mathbf{I}(\delta$ is a small positive constant) \\ & The joint $\mathbf{R L S}$ algorithm (for $n=N, N+1, \ldots) \\ & \quad \frac{\lambda^{-1} \mathbf{R}[n-N] \tilde{\mathbf{g}}_{M L}[n-N]}{1+\lambda^{-1} \tilde{\mathbf{g}}_{M L}^{H}[n-N] \mathbf{R}[n-N] \tilde{\mathbf{g}}_{M L}[n-N]} \\ \mathbf{k}[n]= & \mathbf{W}[n-N] \tilde{\mathbf{g}}_{M L}[n-N] \\ \hat{\mathbf{g}}[n]= & \mathbf{\mathbf { g }}[n]-\hat{\mathbf{g}}[n] \\ \mathbf{e}[n] & \mathbf{W}[n]=N]+\mathbf{k}^{H}[n] \mathbf{e}[n] \\ \mathbf{R}[n]= & \lambda^{-1}\left(1-\mathbf{k}[n] \tilde{\mathbf{g}}_{M L}^{H}[n-N]\right) \mathbf{R}[n-N] \\ & \end{aligned}$

As discussed earlier, UWA systems suffer from inherently long propagation delays, which pose additional challenges in the design of a predictor. To counteract this problem, channel prediction one travel time ahead ( $N$ steps) is achieved by using an RLS predictor of a low order $M$ (e.g. $M=1$ or $M=2$ ) and a small forgetting factor $\lambda$ (e.g. $\lambda=0.5$, which corresponds to an effective window of length $L_{e f f}=$ $1 /(1-\lambda)=2$ ). Note that the forgetting factor $\lambda$ is uniquely specified for all $P$ channel coefficients. With a small order $M$ and only a few significant paths, i.e. a small $P$, computational 


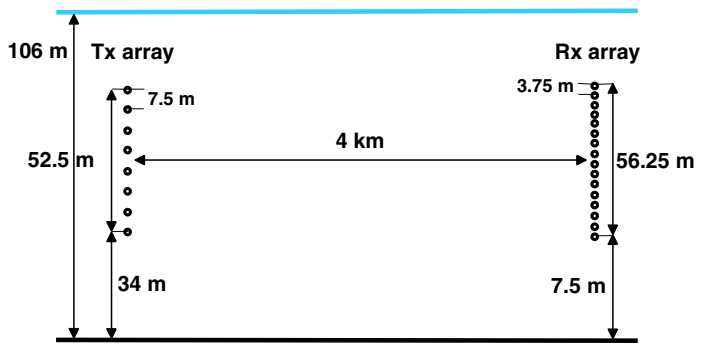

Fig. 3. The geometry of the KAM08 experiment.

complexity of joint channel prediction is sufficiently low to allow for a practical implementation.

The structure of the matrix $\mathbf{W}[n]$ is primarily driven by the geometry of the propagation environment, i.e. not all of the propagation paths are mutually correlated. In the present data set, the strongest arrival often exhibits more stability, and the contribution from the other, weaker paths in its prediction appears to be negligible. Therefore, the strongest path can be predicted independently, without loss in performance. In other words, if the channel coefficient $k$ corresponds to the strongest path, Eq. (19) can be modified as follows: the $k$-th column of $\mathbf{W}[n]$ is recursively updated only for those elements that correspond to the prior observations of the $k$-th coefficient $\left(\tilde{g}_{k}^{l}[n], \tilde{g}_{k}^{l}[n-1], \ldots, \tilde{g}_{k}^{l}[n-M+1]\right)$ at every receive element $l, 0 \leq l \leq L-1$. In contrast, exploiting the correlation between remaining paths may lead to performance improvement, whose exact amount is determined by the environmental profile, and accuracy of the channel estimation and Doppler estimation.

\section{EXPERIMENTAL RESUlts}

In this section we address the performance of the proposed prediction method using real data collected during the KAM08 experiment. The geometry of the experiment is illustrated in Fig 3. Results in this paper are given for the transmissions from the fourth transducer from the bottom $(49.5 \mathrm{~m}$ depth). Our wideband channel probe signal is a Turyn sequence [9] of length 28, BPSK modulated onto a carrier of frequency $f_{c}=16 \mathrm{kHz}$ at a rate of $6250 \mathrm{bps}$. The probe signal is transmitted every $T=155 \mathrm{~ms}$ for a $60 \mathrm{~s}$ transmission interval. The sampling rate is $50 \mathrm{kHz}$. Since propagation delay for one travel time is $2.67 \mathrm{~s}$, and the channel probe is transmitted every $155 \mathrm{~ms}$, our goal is to predict the channel at least $N=17$ steps ahead.

Our first task is to clearly indicate that an RLS algorithm of a small order $M$ and a small forgetting factor $\lambda$ is the right choice for the design of adaptive prediction (10). To do so, we analyze the behavior of the RLS algorithm for different values of $M$ and $\lambda$. In Fig. 4, we compare the mean squared error in the prediction for different $M$ and $\lambda$ for path 0 (see Fig. 1 for path numbering), with single-channel processing at the receive element $\mathrm{Rx}$ 7. The advantage of using channel prediction is apparent for $M=1$ and $\lambda=0.5$, for which a $5 \mathrm{~dB}$ improvement is obtained at the prediction lag of

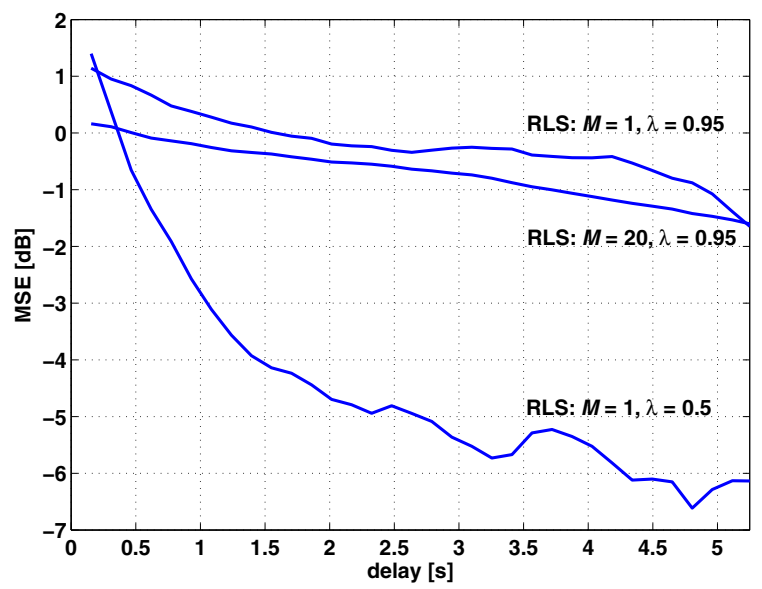

Fig. 4. Performance of the RLS algorithm: Mean squared error (MSE) of the predicted coefficient $\hat{g}_{0}[n+N]$ relative to its outdated estimate $\tilde{g}_{0}[n]$, as a function of prediction lag $N T$. Results are shown for $L=1(\operatorname{Rx} 7)$ and different combinations of the filter size $M$ and the forgetting factor $\lambda$.

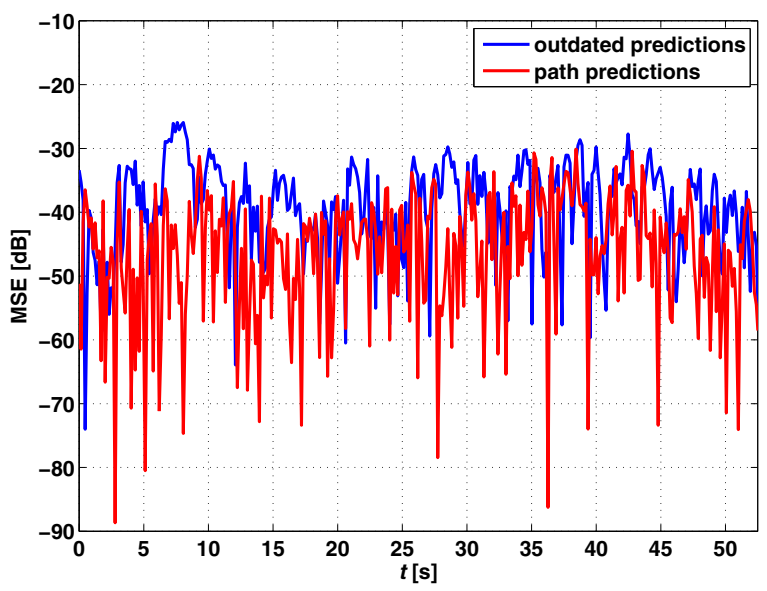

Fig. 5. Performance of the RLS algorithm for path 0 , with $M=1, \lambda=0.5$, $N=17$ and $L=1(\operatorname{Rx} 7)$.

$2.67 \mathrm{~s}$ (propagation delay of the actual system geometry in the experiment). In Fig. 5, we compare the MSE achieved by the prediction $\hat{g}_{0}[n+N]$ with that achieved by an outdated estimate $\tilde{g}_{0}[n]$. Channel prediction is performed independently for each coefficient in this example, i.e. the matrix $\mathbf{W}[n]$ is constrained to be diagonal.

In Fig. 6, we illustrate the benefits of multi-channel processing. The MSE result indicates an obvious advantage when two receive elements are combined to predict desired channel coefficient. Increasing the number of the receive elements for multi-channel processing further improves the performance of channel prediction.

In Table II, we provide the MSE achieved by joint channel path prediction relative to the individual prediction. We assume single-channel processing $(L=1)$. We present results for 6 different elements from the receiver array - Rx 7, 8, 9, 10, 


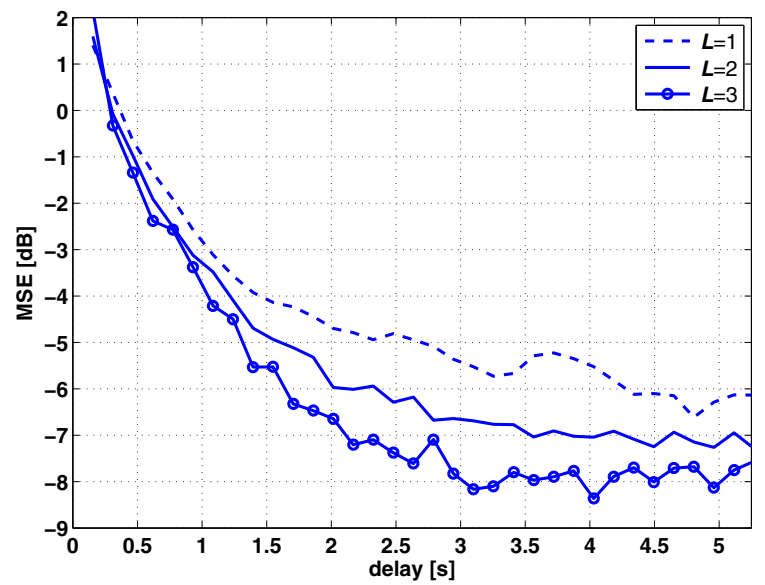

Fig. 6. Performance of the RLS algorithm for path 0 , with $M=1$, $\lambda=0.5$ and $N=17$. Single-channel processing $(\mathrm{L}=1)$ uses $\mathrm{Rx} 7$; twochannel processing uses Rx 7 and Rx 16; three-channel processing uses Rx 7 , Rx 10 and Rx 16.

13 and 16 ( $\mathrm{Rx} 16$ is the closest element to the ocean surface). With $\mathrm{Rx} 7$ and $\mathrm{Rx} 8, P=4$ significant paths are identified; with $\operatorname{Rx} 9$ and $\operatorname{Rx} 10, P=3$, and with $\operatorname{Rx} 13$ and $\operatorname{Rx} 16$, $P=2$. For example, with Rx 7 , the second row represents the case in which $g_{0}(t)$ and $g_{2}(t)$ are predicted jointly, while $g_{1}(t)$ and $g_{3}(t)$ are predicted independently. Results are given for four channel paths identified as significant. Blank cells in the table indicate that the corresponding paths are not combined for joint prediction. This combination yields a $2.47 \mathrm{~dB}$ improvement in the prediction of $g_{0}(t)$, but $1.07 \mathrm{~dB}$ degradation in the prediction of $g_{2}(t)$. Joint prediction of all four paths does not result in any improvement, except for $g_{3}(t)$, indicating that not all of the propagation paths are mutually correlated. Joint estimation of paths 0 and 3 , which are the weak ones (see Fig. 1), yields a good improvement when these paths are combined with path 2 , with the corresponding reductions in the MSE of $2.47 \mathrm{~dB}$ and $4.98 \mathrm{~dB}$, respectively. Path 1 appears to be fairly independent from the rest of the channel impulse response.

These results indicate that there is a certain advantage to joint path prediction. More importantly, our results show that channel prediction, whether conducted individually or jointly for the significant paths, is possible even in the presence of long acoustic delays, and that it offers an improvement over an ignorant approach that uses outdated channel estimates. This fact offers a motivation to pursue adaptive modulation as a means of achieving improved performance over time-varying UWA channels.

\section{CONCLUSiOns}

In this paper we investigated the possibility of predicting an UWA channel at least one travel time ahead. The key step in providing a stable reference for channel prediction is compensation of the motion-induced phase offset. Together with motion compensation, a matching pursuit algorithm is
TABLE II

RELATIVE MSE (IN DECIBELS) FOR $N=17$. BOLDFACE NUMBERS CORRESPOND TO CASES IN WHICH JOINT PREDICTION YIELDS AN IMPROVEMENT.

\begin{tabular}{|c|c|c|c|c|c|}
\hline \multicolumn{3}{|c|}{$\overline{\mathrm{Rx} 9}$} & \multicolumn{3}{|c|}{$\mathrm{Rx} 10$} \\
\hline$g_{0}$ & $g_{1}$ & $\underline{g_{2}}$ & $g_{0}$ & $g_{1}$ & $g_{2}$ \\
\hline 1.94 & 0.14 & 0.15 & 2.99 & 0.35 & -5.09 \\
\hline 1.48 & -1.10 & & 1.61 & $\begin{array}{l}-1.88 \\
\end{array}$ & \\
\hline \multirow[t]{2}{*}{0.44} & & -0.13 & 1.48 & & $\begin{array}{l}-7.34 \\
\end{array}$ \\
\hline & -1.19 & 0.54 & & -0.15 & -1.55 \\
\hline \multicolumn{4}{|c|}{$\overline{\mathrm{Rx} 8}$} & \multicolumn{2}{|c|}{ Rx 13 } \\
\hline$g_{0}$ & $g_{1}$ & $g_{2}$ & $g_{3}$ & $g_{0}$ & $g_{1}$ \\
\hline 1.76 & $\overline{0.28}$ & $\overline{0.69}$ & $\overline{0.96}$ & $\overline{1.08}$ & -2.08 \\
\hline \multirow[t]{3}{*}{-0.64} & -0.24 & & & & \\
\hline & -0.81 & -1.21 & & & \\
\hline & -0.37 & & -1.57 & & \\
\hline \multicolumn{4}{|c|}{ Rx 7} & \multicolumn{2}{|c|}{ Rx 16} \\
\hline$g_{0}$ & $g_{1}$ & $g_{2}$ & $g_{3}$ & $g_{0}$ & $g_{1}$ \\
\hline 0.03 & 1.29 & 2.68 & -3.16 & 1.42 & -2.11 \\
\hline \multirow[t]{3}{*}{-2.47} & & 1.07 & & & \\
\hline & -1.34 & 0.26 & & & \\
\hline & & 1.07 & -4.94 & & \\
\hline
\end{tabular}

used to identify the significant path coefficients (there may only be a few). The estimated path coefficients are finally processed by an adaptive RLS predictor, which is designed to have a low order and short memory to account for large prediction lags (long feedback delays). The predictor is implemented in a multi-channel configuration, and allows for either joint or individual treatment of the path coefficients. Experimental results clearly indicate the superiority of the proposed prediction method as compared to the simple use of outdated channel estimates. The apparent feasibility of predicting the channel one or more travel times ahead leads us to conclude that adaptive modulation methods may be viable for reliable, high-rate UWA communications.

\section{REFERENCES}

[1] M. Stojanovic, "Underwater Acoustic Communications: Design Considerations on the Physical Layer," IEEE/IFIP Fifth Annual Conference on Wireless On demand Network Systems and Services (WONS 2008), Garmisch-Partenkirchen, Germany, Jan. 2008.

[2] A. J. Goldsmith and S.-G. Chua, "Variable-Rate Variable-Power MQAM for Fading Channels," IEEE Trans. Comm., vol. 45, no. 10, pp. 12181230, Oct. 1997.

[3] D. L. Goeckel, "Adaptive Coding for Time-Varying Channels Using Outdated Fading Estimates," IEEE Trans. Comm., vol. 47, no. 6, pp. 844-855, June 1999.

[4] M. Chitre, M. Stojanovic, S. Shahabudeen and L. Freitag, "Recent Advances in Underwater Acoustic Communications \& Networking," in Proc. IEEE Oceans'08 Conference, Quebec City, Canada, Sept. 2008.

[5] P. Qarabaqi and M. Stojanovic, "Statistical Modeling of a Shallow Water Acoustic Communication Channel," Proc. Underwater Acoustic Measurements Conference, Nafplion, Greece, June 2009.

[6] J. G. Proakis and D. K. Manolakis, Digital Signal Processing, 4th ed. Prentice Hall, 2006.

[7] A. Radosevic, J. G. Proakis and M. Stojanovic, "Statistical Characterization and Capacity of Shallow Water Acoustic Channels," Proc. IEEE Oceans'09 Conference, Bremen, Germany, May 2009.

[8] W. Li and J. Preisig, "Estimation of Rapidly Time-Varying Sparse Channels," IEEE J. Ocean. Eng., vol. 32, no. 4, pp. 927-939, Oct. 2007.

[9] R. J. Turyn, "Sequences with Small Correlation," In Error Correcting Codes: Proceedings of a Symposium (H. B. Mann, ed.), New York: Wiley, pp. 195-228, 1968. 